\title{
CFD SIMULATION OF A STIRRED DISHED BOTTOM VESSEL
}

\author{
Petr VlČeK, Jan SkočIlas*, Tomáš Jirout \\ Czech Technical University in Prague, Faculty of Mechanical Engineering, Department of Process Engineering, \\ Technicka 4, 16607 Prague 6, Czech Republic \\ * corresponding author: jan.skocilas@fs.cvut.cz
}

\begin{abstract}
This paper deals with simulation of the fluid flow in a stirred curved-bottom vessel equipped with three curved blade impellers. The power number and the impeller flow rate number are dimensionless characteristics of the system determined from simulation results and compared with relevant experimental data or data from the literature. The model of the system was created in the conventional Gambit and Fluent program. The system is solved for two designs - for an unbaffled vessel, and for a baffled vessel. The vessel is filled with water and the impeller speed is $100 \mathrm{~min}^{-1}$.

Three turbulent models were used for the solution: $\mathrm{k}-\varepsilon, \mathrm{k}-\omega$ and RSM. The results were compared with experimental data or data from the literature. The $\mathrm{k}-\varepsilon$ model had the smallest demands on processor time, and the results compared satisfactorily with the experimental data. The model provides comprehensive information about the characteristics of the system.
\end{abstract}

KEYWORDS: CFD, FLUENT, mixing, curved bottom, stirred vessel.

\section{INTRODUCTION}

The main requirements to be met by an engineer specializing in the design of new equipment or in analyzing and optimizing existing equipment are to minimize the time taken to solve the assigned problem and to minimize the cost of solving the problem. When there is no contemporary equivalent of the required equipment, and when design verification experiments are too expensive, a cheap and quick alternative is to make simulations of the processes and equipment. Mathematical models based on fundamental physical principles, and even on empirics that can predict the behaviour of the system with sufficient (engineering) accuracy, provide a modern and robust tool for developers and for other users. However, the results of the numerical simulations generated by mathematical models need to be verified experimentally or by approximate analytical calculations. If the prediction corresponds with the experimental data or with calculations, the model of the virtual equipment becomes a powerful tool that enables various options of structural or technological modifications of the equipment to be checked easily, without expensive prototyping and experimentation. An advantage of applying a verified equipment model is that the design of a series of pieces of equipment of the same type but with various dimensions can be enhanced. This is referred to as scale up, and is a typical example of the production program of engineering companies that specialize in a single specific branch of industry.

This study deals with simulation of the flow in stirred vessels. The objectives of the paper are to determine complex dimensionless characteristics power number and impeller flow rate number - by carrying out investigations of the velocity field within the stirred vessel using a simulation of the equipment.
The aim is to propose a model that will predict the value of dimensionless characteristics with sufficient accuracy (in comparison with experimental data). Then this model can be used to determine the characteristics of the system, e.g. with a different rotation speed or with a different distance of the impeller from the vessel bottom. The system will be solved for two designs - for an unbaffled vessel and for a baffled vessel. The vessel is filled with water, and the impeller speed is set to $100 \mathrm{~min}^{-1}$.

\section{Problem ANALYSiS}

In terms of flow simulation, a stirred vessel is a special case of the flow problem (without any inlets or outlets), and it requires a slightly different approach from usual types of simulated processes, e.g. fluid flow in a channel, body circumfluence, or mixing of two fluids in a pipe or in a nozzle. In our case, it is necessary to ensure the movement of the impeller in the tank, and to adjust the boundary conditions of the problem (the volume of liquid is constant; the system has no inflows or outflows). Our task is to determine the torque acting on the blades of the impeller and the impeller flow rate, the power number and the impeller flow rate number. These parameters can be defined by equations (1) and (2).

Power number

$$
P_{0}=\frac{2 \pi M_{K}}{\varrho n^{2} d^{5}}
$$

where $M_{K}$ is the torque acting on the impeller $[\mathrm{N} \cdot \mathrm{m}]$, $\varrho$ is the density of the liquid $\left[\mathrm{kg} \cdot \mathrm{m}^{3}\right], n$ is the rotation speed of the impeller $\left[\mathrm{s}^{-1}\right]$, and $d$ is the diameter of the impeller $[\mathrm{m}]$. 


\begin{tabular}{cccccccccc}
\hline$D$ & $H / D$ & $H_{2}$ & $D / d$ & $h / d$ & $t / d$ & $d$ & $R$ & $h$ & $t$ \\
\hline $300 \mathrm{~mm}$ & 1 & $15 \mathrm{~mm}$ & 2 & 0.15 & 0.03 & $150 \mathrm{~mm}$ & $50 \mathrm{~mm}$ & $22.5 \mathrm{~mm}$ & $5 \mathrm{~mm}$ \\
\hline
\end{tabular}

TABLE 1. Geometrical parameters and dimensions of the vessel and the impeller with curved blades.
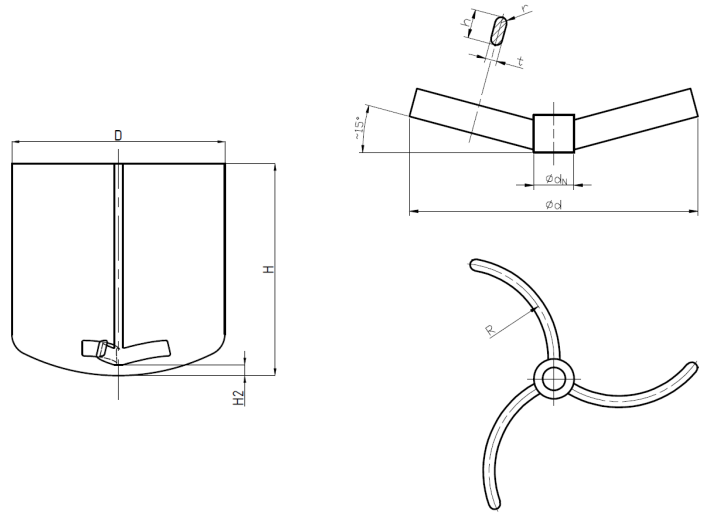

Figure 1. Scheme and geometry of the impeller and the vessel.

Impeller flow rate number

$$
N_{Q}=\frac{Q}{n d^{3}},
$$

where $Q$ is the impeller pumping capacity of the fluid $\left[\mathrm{m}^{3} \cdot \mathrm{s}^{-1}\right]$.

In terms of mathematical formulations, only NavierStokes equations together with the continuity equation will be resolved in the flow of a Newtonian fluid in a vessel. The flow regime of the liquid in the vessel has to be verified with respect to the specified parameters. A simple calculation of the Reynolds number will determine the flow regime.

Reynolds number for stirred vessels

$$
R e=\frac{n d^{2} \varrho}{\mu} \approx 37500,
$$

where the working fluid is water, $\mu$ is the dynamic viscosity $[\mathrm{Pa} \cdot \mathrm{s}]$, and the impeller speed is $100 \mathrm{~min}^{-1}$.

The calculated Reynolds number value indicates that the flow regime is turbulent.

We will use the maximum simplification of the model that is compatible with obtaining relevant results comparable with the experimental data. We will consider a 3D geometric model for the steady flow regime. Due to the Reynolds number value, we consider a turbulent flow turbulence model. We use RANS models for a quick analysis of the problem and to obtain the required outputs (global dimensionless characteristics of the system). For the same reason, we will simulate the interaction between the stirrer and the vessel wall using the MRF approach. We also neglect the formation of the central vortex at the water level, because the rotation speed of the impeller $100 \mathrm{~min}^{-1}$ is low, and the vortex that forms is minimal (verified by experiment). Thus, we use symmetry for the water level boundary condition.
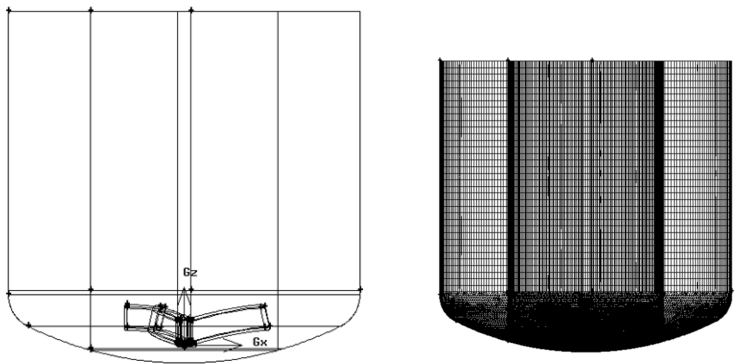

Figure 2. Final layout of the geometric model with the sub-volumes and a grid for the unbaffled vessel.

\section{MODEL GEOMETRY AND GRID}

The basic geometry of the model was created in the Autodesk Inventor 3D Modeller. The unbaffled vessel, the baffled vessel and the impeller geometry were created separately. The impeller (manufactured by TENEZ company) has three curved blades with rounded edges. The baffled vessel is equipped with four baffles. The vessel has a curved bottom. The basic dimensions are shown in Figure 1 and Table 1 . Appropriate models were combined into the required model in the Gambit 2.4 program [6]. The resulting volume was subsequently divided into rotating and stationary parts, as required by the MRF method. The model was further decomposed into sub-volumes to form an unstructured grid, see Figure 2, which completely describes our stirred vessel. The model contains 2.5 million cells for a system without baffles and 3.1 million cells for a system with baffles.

\section{Processor Set UP AND CALCULATION}

The solutions for the two cases with the unbaffled vessel and the baffled vessel were carried out in FLUENT v14.0 [5]. The solver was adjusted according to Table 3 .

The final number of iterations of the calculation for $\mathrm{k}-\varepsilon$ and $\mathrm{k}-\omega$ was approximately 15000 for the first calculation with adjusted lower accuracy, and then 20000 iterations to calculate with adjusted greater accuracy. The average computation time was 48 hours when using eights parallel computing threads. For RSM, the total number of iterations was 50000 and the computation time was four days. A total of six models were calculated - the unbaffled vessel with three turbulence models, and the baffled vessel with three turbulence models.

The power number was calculated from the torque acting on the impeller. The torque was determined 


\begin{tabular}{|c|c|c|c|c|c|}
\hline \multicolumn{3}{|c|}{ Unbaffled vessel } & \multicolumn{3}{|c|}{ Baffled vessel } \\
\hline $\begin{array}{l}\text { Element } \\
\text { type }\end{array}$ & $\begin{array}{c}\text { Number of } \\
\text { elements }\end{array}$ & $\begin{array}{l}\text { Proportion } \\
\text { of elements }\end{array}$ & $\begin{array}{l}\text { Element } \\
\text { type }\end{array}$ & $\begin{array}{l}\text { Number of } \\
\text { elements }\end{array}$ & $\begin{array}{l}\text { Proportion } \\
\text { of elements }\end{array}$ \\
\hline Hexahedron & 611000 & $24.7 \%$ & Hexahedron & 1032000 & $33.2 \%$ \\
\hline Pyramid & 37000 & $1.5 \%$ & Pyramid & 45000 & $1.5 \%$ \\
\hline Tetrahedron & 1822000 & $73.8 \%$ & Tetrahedron & 2027000 & $65.3 \%$ \\
\hline Summary & 2471000 & $100 \%$ & Summary & 3104000 & $100 \%$ \\
\hline
\end{tabular}

TABLE 2. Number of elements in the grid of the stirred vessel model.

\begin{tabular}{lc}
\hline Flow regime & Turbulent \\
\hline Turbulence models & $\mathrm{k}-\omega, \mathrm{k}-\varepsilon, \mathrm{RSM}$ \\
\hline Fluid in the stirred vessel & water \\
\hline Boundary conditions & $\begin{array}{c}\text { rotation } \mathrm{MRF}, \\
\text { rotation speed } 100 \mathrm{~min}^{-1}\end{array}$ \\
\hline Under relaxation factors & Default \\
\hline Discretization - pressure & Second order \\
\hline Discretization - momentum & Second order upwind \\
\hline Discretization - turbulent kinetic energy & Second order upwind \\
\hline Discretization - turbulent dissipation rate & Second order upwind \\
\hline Discretization - Reynolds stresses & Second order upwind \\
\hline Residuals & $10^{-4}$ \\
\hline
\end{tabular}

TABLE 3. Solver setting for the stirred vessel.

directly from Fluent software using the in-built function which calculates the torque from the shear stress acting on the user selected surface. The torque acting on the shaft was neglected due to the small radius of the shaft. The impeller flow rate number was evaluated from impeller pumping capacity determined by integrating the velocity field on the surface located above (or below) the impeller (the radial flow rate is neglected). This surface is circular in shape with a radius that is one millimetre greater than impeller, and it was formed together with the geometry and the mesh.

\section{Simulation Results - POSTPROCESSOR}

This section presents the results of two types of stirred vessels with an agitator for various models of turbulence. First, we will concentrate on comparing the distribution of the monitored quantities in the crosssections of the vessel for first and second order accuracy. The comparison of the solution results for the unbaffled stirred vessel using the $\mathrm{k}-\varepsilon$ model is successively shown in Figures 3 to 6 . The results of the calculation of first order accuracy are always shown on the left side, and the results for second order of accuracy are shown on the right side. There is a com- parison of the static pressure contours in the vessel and the velocity magnitude contours.

The figures show that when a higher order of accuracy is used the contours of the parameters are smoother and some instability fields are suppressed, see for example the velocity distribution in the vessel viewed in vertical section in Figure 5, e.g. the area in the vicinity of the wall in the transition between the cylindrical part and an arched part of the vessel.

Three turbulent models were used for the solution. The results are compared in the following figures for the contours of the velocity magnitude. All figures are presented for a calculation of second order accuracy.

There are no significant differences in the results obtained from all the turbulence models. The maximum and minimum values are also at least of the same order (except RSM, where the maximum pressure is four times higher).

A better overall comparison of turbulence model predictions can be obtained with the measured data, especially the calculated power numbers and impeller flow rate numbers. See Tables 4 to 7

The stated value for the pump power $N_{\mathrm{QE}}=0.3 \mathrm{was}$ obtained for a Pfaudler impeller from the literature [4] (not for the investigated geometry of an impeller). However the system geometry (flat or curved bottom vessel, impeller distance from bottom, etc.) corre- 

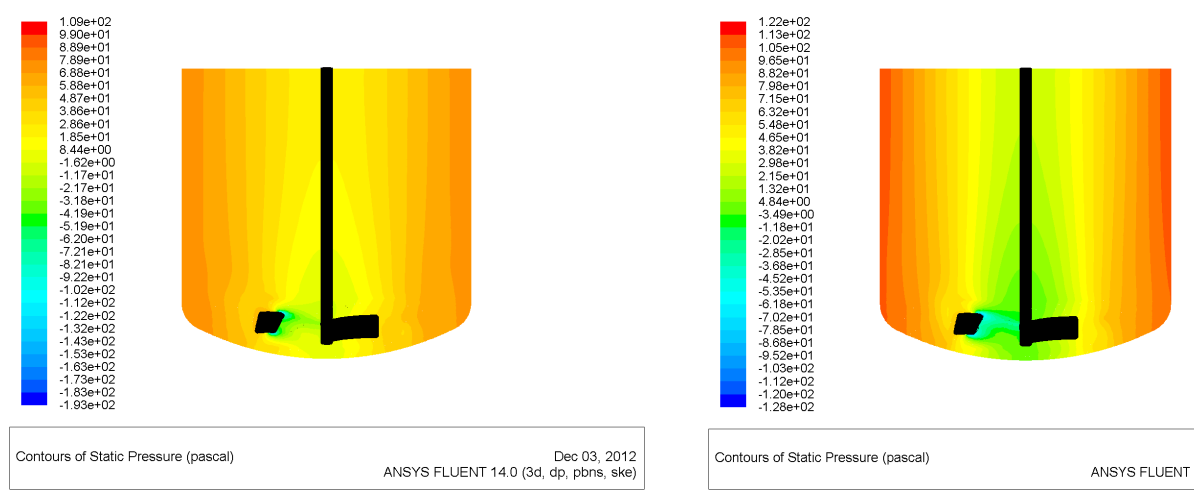

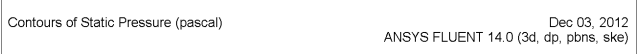

Figure 3. Unbaffled vessel. Vertical cut of the vessel. Static pressure contours in the vessel. Left - first order accuracy, right — second order accuracy.
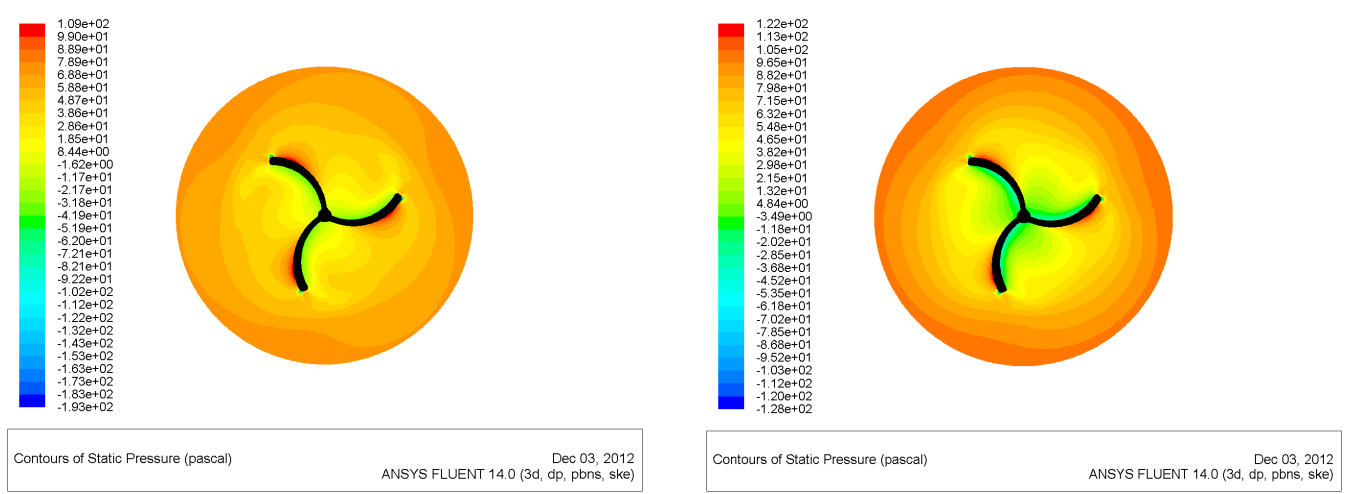

Figure 4. Unbaffled vessel. Horizontal cut of the vessel in the plane of the impeller. Static pressure contours in the vessel. Left - first order accuracy, right — second order accuracy.
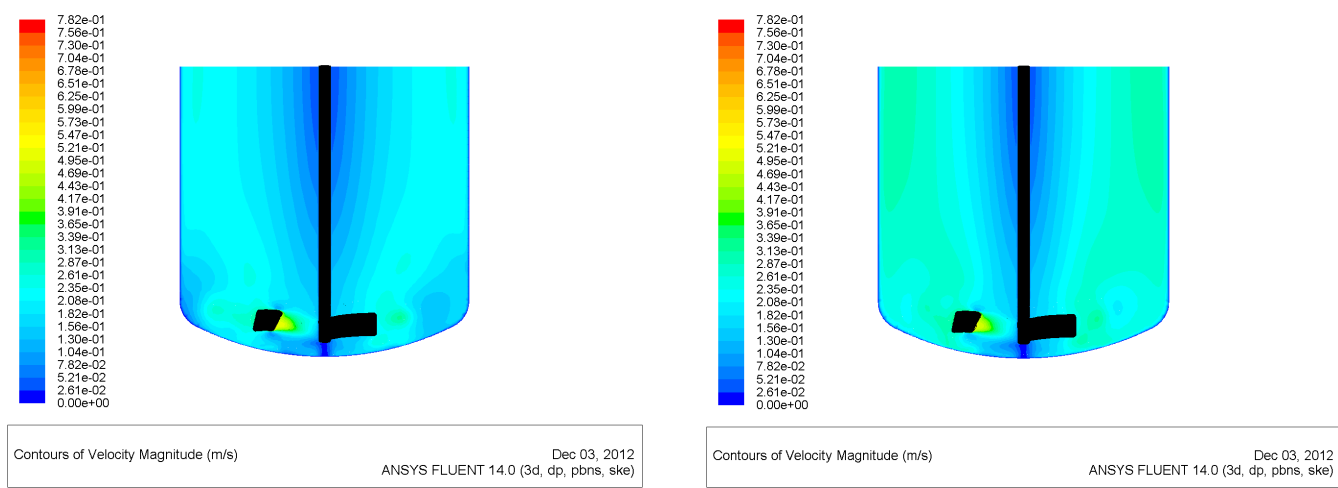

FIGURE 5. Unbaffled vessel. Vertical cut of the vessel. Velocity magnitude contours in the vessel. Left — first order accuracy, right - second order accuracy. $\mathrm{k}-\varepsilon$ model.
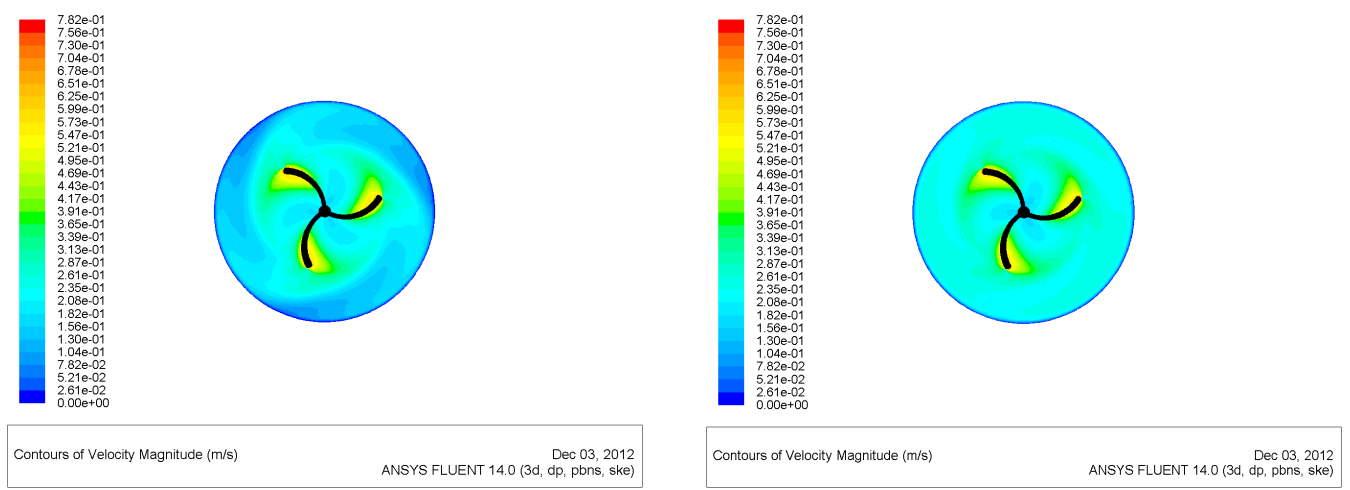

Figure 6. Unbaffled vessel. Horizontal cut of the vessel in the plane of the impeller. Velocity magnitude contours in the vessel. Left — first order accuracy, right — second order accuracy. k- $\varepsilon$ model. 

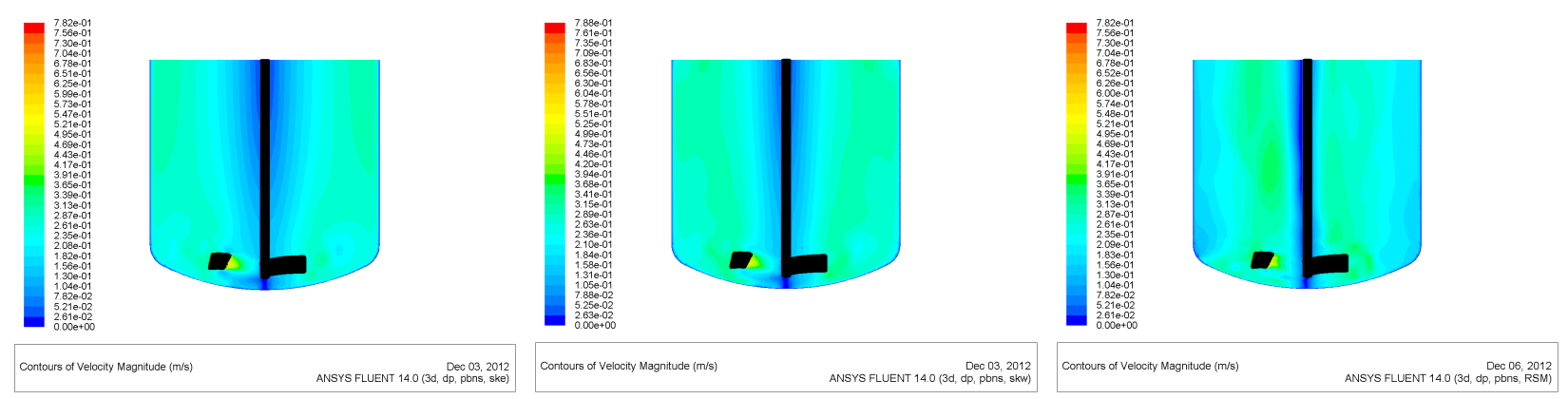

Figure 7. Unbaffled vessel. Vertical cut. Contours of velocity magnitude. Three turbulence models: $\mathrm{k}-\varepsilon, \mathrm{k}-\omega$ and RSM.
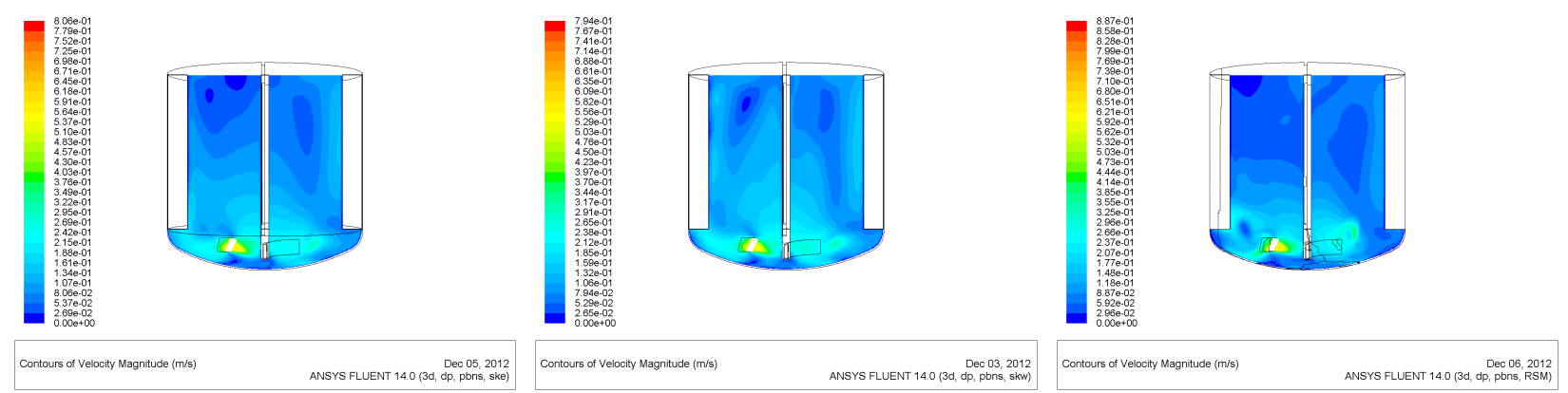

Figure 8. Baffled vessel. Vertical cut. Contours of velocity magnitude. Three turbulence models: k- $\varepsilon$, k- $\omega$ and RSM.
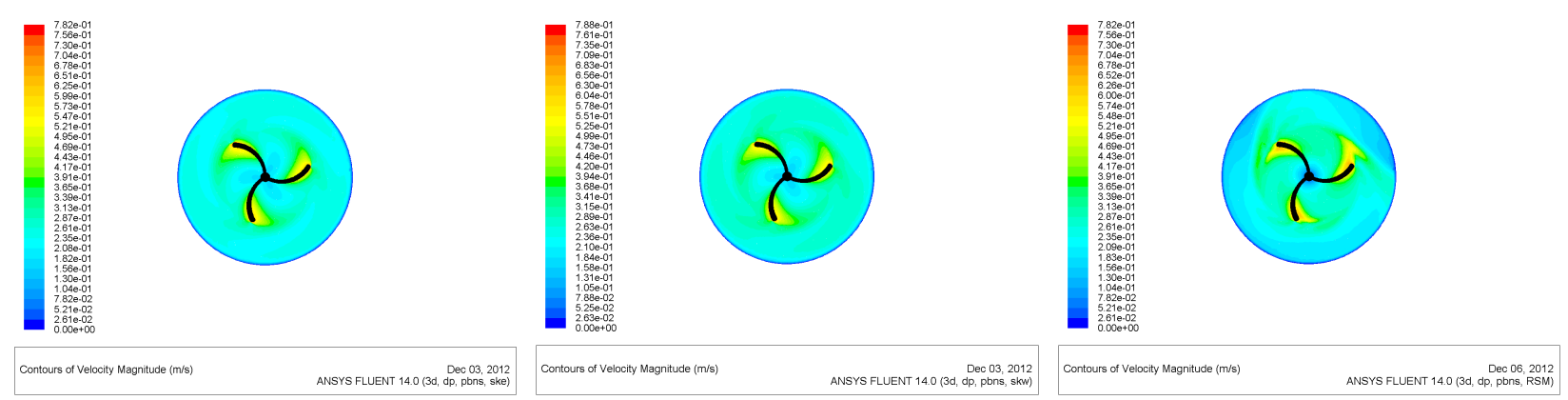

Figure 9. Unbaffled vessel. Horizontal cut in the plane of the impeller. Contours of velocity magnitude. Three turbulence models: $\mathrm{k}-\varepsilon, \mathrm{k}-\omega$ and RSM.
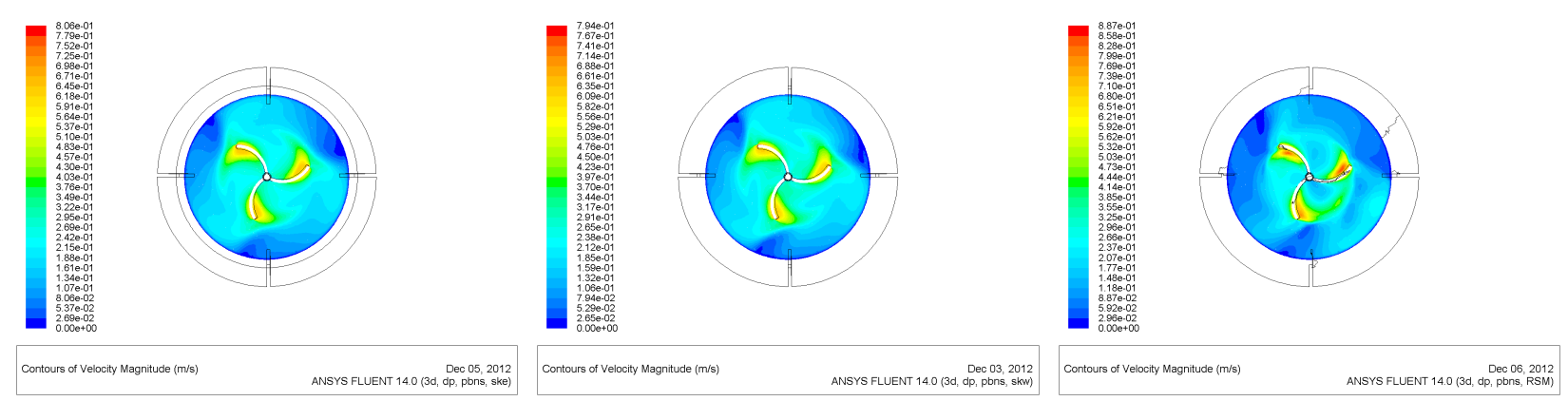

Figure 10. Baffled vessel. Horizontal cut in the plane of the impeller. Contours of velocity magnitude. Three turbulence models: $\mathrm{k}-\varepsilon, \mathrm{k}-\omega$ and RSM. 


\begin{tabular}{ccccccc}
\hline & $N_{\mathrm{QC}}$ & $N_{\mathrm{QE}}$ & Deviation & $P_{\mathrm{OC}}$ & $P_{\mathrm{OE}}$ & Deviation \\
\hline $\mathrm{k}-\varepsilon$ & 0.23 & 0.3 & $23 \%$ & 0.56 & 0.46 & $-22 \%$ \\
\hline $\mathrm{k}-\omega$ & 0.19 & 0.3 & $37 \%$ & 0.51 & 0.46 & $-11 \%$ \\
\hline $\mathrm{RSM}$ & 0.21 & 0.3 & $30 \%$ & 0.43 & 0.46 & $7 \%$ \\
\hline
\end{tabular}

TABlE 4. Comparison of the power numbers and the impeller flow rate number for the unbaffled stirred vessel, first order discretization. Indexes: $\mathrm{C}$ - calculated, $\mathrm{E}$ - measured.

\begin{tabular}{ccccccc}
\hline & $N_{\mathrm{QC}}$ & $N_{\mathrm{QE}}$ & Deviation & $P_{\mathrm{OC}}$ & $P_{\mathrm{OE}}$ & Deviation \\
\hline $\mathrm{k}-\varepsilon$ & 0.19 & 0.3 & $37 \%$ & 0.52 & 0.46 & $-13 \%$ \\
\hline $\mathrm{k}-\omega$ & 0.16 & 0.3 & $47 \%$ & 0.51 & 0.46 & $-11 \%$ \\
\hline $\mathrm{RSM}$ & 0.12 & 0.3 & $60 \%$ & 0.30 & 0.46 & $35 \%$ \\
\hline
\end{tabular}

TABlE 5. Comparison of the power numbers and the impeller flow rate number for the unbaffled stirred vessel, second order discretization. Indexes: $\mathrm{C}$ - calculated, $\mathrm{E}$ - measured

\begin{tabular}{ccccccc}
\hline & $N_{\mathrm{QC}}$ & $N_{\mathrm{QE}}$ & Deviation & $P_{\mathrm{OC}}$ & $P_{\mathrm{OE}}$ & Deviation \\
\hline $\mathrm{k}-\varepsilon$ & 0.43 & 0.3 & $-43 \%$ & 0.79 & 1.4 & $44 \%$ \\
\hline $\mathrm{k}-\omega$ & 0.38 & 0.3 & $-27 \%$ & 0.75 & 1.4 & $46 \%$ \\
\hline $\mathrm{RSM}$ & 0.32 & 0.3 & $-7 \%$ & 0.71 & 1.4 & $49 \%$ \\
\hline
\end{tabular}

TABLE 6. Comparison of the power numbers and the impeller flow rate number for the baffled stirred vessel, first order discretization. Indexes: $\mathrm{C}$ - calculated, E - measured

\begin{tabular}{ccccccc}
\hline & $N_{\mathrm{QC}}$ & $N_{\mathrm{QE}}$ & Deviation & $P_{\mathrm{OC}}$ & $P_{\mathrm{OE}}$ & Deviation \\
\hline $\mathrm{k}-\varepsilon$ & 0.44 & 0.3 & $-47 \%$ & 0.81 & 1.4 & $42 \%$ \\
\hline $\mathrm{k}-\omega$ & 0.42 & 0.3 & $-40 \%$ & 0.85 & 1.4 & $39 \%$ \\
\hline $\mathrm{RSM}$ & 0.31 & 0.3 & $-3 \%$ & 0.61 & 1.4 & $56 \%$ \\
\hline
\end{tabular}

TABLE 7. Comparison of the power numbers and the impeller flow rate number for the baffled stirred vessel, second order discretization. Indexes: $\mathrm{C}-$ calculated, $\mathrm{E}-$ measured.

sponding to the stated value was not described. The place at which of the flow rate was determined is also not clear. The $N_{\mathrm{QC}}$ values therefore serve rather for mutual comparison of the model. The power number $P_{\mathrm{O}}$ serves better for comparing the results with measured values, because we investigated them directly. The results of models $\mathrm{k}-\varepsilon$ and $\mathrm{k}-\varepsilon$ are comparable, but the RSM model predicts mostly lower values of the parameters, especially for second order accuracy of the discretization. The reasons for obtaining different results with the RSM model were obviously problems with the convergence of the simulations. Especially when second-order discretization accuracy was used, the residues and the flow rate fluctuated significantly, and so the flow rate value is determined by the consideration given by the authors to stabilisation of the calculation. In addition, the RSM model requires more computational time for the solution than models $\mathrm{k}-\varepsilon$ and $\mathrm{k}-\varepsilon$. Model $\mathrm{k}-\varepsilon$ provided slightly smaller deviations of the power numbers for the second order, but this model had small oscillations of the residues and therefore also had more problems with convergence than $\mathrm{k}-\varepsilon$.

On the basis of these conclusions, we recommend using the robust $\mathrm{k}-\varepsilon$ model, which made the smallest demands on processor time, and the results were sufficiently comparable with the experimental data when second order discretization accuracy was used. There are more deviations of the power numbers for a baffled vessel, but the predictions of the models are similar. This difference is probably due to the difference in the shape of the end of the stirrer blades, where the most power dissipation takes place. The impeller for which the power number was determined experimentally has a straight end of the blades, while the model for the simulation has an oblique end of the blades, so that the model impeller has a smaller blade area. The difference is in the largest radius. The dissipated power depends on the fifth power of the radius, and the predicted power numbers for all models are therefore smaller than the experimentally-derived value. 


\section{Conclusion}

- Our model provides complex information about the integral characteristics of the system. The values of the parameters agree with the experimental findings in terms of orders of magnitude. The distribution of the pressure, the velocity and the other variables can be regarded as credible. In particular, the results for the unbaffled vessel can be considered suitable for engineering design, where the deviation is around 10 per cent.

- This model can be directly used for calculating the flow characteristics inside the vessel for various rotation speeds. The model can also be used to predict the distribution of characteristics dependent on various geometrical configurations - impeller displacement from the bottom of the vessel, more baffles or a different layout of the baffles, etc. When there is a change in the geometry, it is necessary to reconstruct the geometric model and the grid of finite volumes, which requires a considerable investment of time. However, if we apply exactly the same procedure for creating a network as for setting up the simulations, the results of this new model can be considered to be at least within the same value order.

- However this model has limitations that must be kept in mind: the boundary condition for the fluid surface inside the vessel; the type of boundary conditions, symmetry, neglecting the effect of the central vortex, which increases as the speed of the impeller rises, or as the Reynolds number rises.

\section{LIST OF SYMBOLS}

$D$ Vessel diameter $[\mathrm{m}]$

$d$ Impeller diameter [m]

$H$ Height of fluid surface [m]

$\mathrm{H}_{2}$ Impeller distance from the bottom [m]

$h$ Height of the impeller blade [m] $t \quad$ Thickness of the impeller blade [m]

$R$ Radius of the curvature blades [m]

$M_{K}$ Torque [N m]

$n$ Rotation speed of the impeller $\left[\mathrm{s}^{-1}\right]$

$Q$ Impeller pumping capacity $\left[\mathrm{m}^{3} \mathrm{~s}^{-1}\right]$

$N_{Q}$ Impeller flow rate number [-]

$P_{O}$ Power number [-]

Re Reynolds number [-]

$\varrho$ Density $\left[\mathrm{kg} \mathrm{m}^{-3}\right]$

$\mu$ Dynamic viscosity $[\mathrm{Pas}]$

\section{ACKNOWLEDGEMEnTs}

This research was supported by the Technology Agency of the Czech Republic under grant TA02011251 "Optimization of enameled mixing equipment according to the technological needs of end users".

\section{REFERENCES}

[1] Wilcox D.C., Aiemo-Obeng V.A., and Kresta S.M.: Turbulence Modeling for CFD: science and practice. 1st Ed. California: DCW Industries, Inc., 1993, pp 460. ISBN 09-636-0510-0.

[2] Joshi J.B., Nere N.K., Rane Ch.V., Murthy B.N, Mathpati C., Patwardham A.W. and Ranade V.V.: CFD simulation of stirred tank: Comparison of turbulent models. Part II:Axial flow impellers, multiple impellers and multiphase dispersion, Can. J. Chem. Eng., 89, 2011, pp. 754-816.

[3] Ceres D., Jirout T., Rieger F.: Mixing of suspensions with curved blade turbine. Inzynieria $i$ aparatura chemiczna, 50, 2011, pp. 7-8. ISSN 0368-0827.

[4] Paul E.L., Aiemo-Obeng V.A., and Kresta S.M. Handbook of industrial mixing: science and practice. Hoboken, N.J.: Wiley-Interscience, 2004, 1377 p. ISBN 04-712-6919-0.

[5] Fluent Inc., 2008. FLUENT 6.3 User's Guide.

[6] Fluent Inc., 2004. Gambit 2.4 Tutorial Guide. 